\title{
LA CONCEPCIÓN ESTRUCTURAL DE LA FÁBRICA EN LA ARQUITECTURA
}

\author{
(STRUCTURAL ANALYSIS OF MASONRY ELEMENTS IN ARCHITECTURE)
}

\author{
Antonio J. Mas Guindal \\ Profesor Titular, Dr. Arquitecto. Universidad Politécnica de Madrid. Dpto. Estructuras de la Edificación. Escuela Técnica Superior \\ de Arquitectura (ETSAM)
}

Fecha de recepción: 18-XII-05

ESPAÑA

\section{RESUMEN}

El presente artículo establece el punto de vista necesario, a juicio del autor, para analizar una estructura de fábrica desde su funcionamiento. Toda la historia de la arquitectura hasta el siglo XIX ha girado en torno a las estructuras de fábrica en todos sus estilos. La introducción de los materiales tenaces (hierro, fundición y acero) con la revolución industrial y el concepto de rigidez como método de análisis compatible, han introducido, tanto en la formación como en la práctica profesional, métodos de análisis basados en la rigidez elástica (relación tensión/ deformación). Las fábricas no pueden ser analizadas desde estos supuestos, una piedra o un ladrillo no tiene una elasticidad fácilmente mensurable, y las fábricas se tornan así como meros agregados de sólidos de trabajo a monotensión (compresión) con enlaces de infinitas articulaciones que les hacen obedecer al modelo de estructuras hiperestáticas de orden infinito, y que además, como todas las demás estructuras, mutan en el tiempo, donde cualquier modelo de análisis puede resultar baladí sino se tienen en cuenta los estados reales (fisurados o agrietados) de la estructura en cada momento de su vida útil.

Este escrito intenta modestamente clarificar los supuestos del análisis, valorando los principios básicos de equilibrio, únicos válidos en el análisis y las condiciones en las que este equilibrio puede realizarse. Los antiguos constructores se valieron de la experiencia como único justificante del equilibrio, para ellos, la única seguridad consistía en que la estructura no colapsaba. Hoy el álgebra vectorial, la observación afinada por instrumentación de precisión, y la determinación a priori de las situaciones de rotura (colapso), permiten realizar conjeturas más aproximadas, pero ciertas, sobre el funcionamiento de las estructuras de fábrica con un nivel de precisión no inferior a sofisticados métodos de análisis, estableciendo de forma tangible niveles de seguridad.

\section{SUMMARY}

The present article establishes the vantage required, in the author's view, to analyze masonry structure performance. Until the nineteenth century, the entire history of architecture revolved around the various styles of such structures. The introduction of high toughness materials (cast iron and steel) with the industrial revolution and the adoption of rigidity as a parameter for compatible analysis laid the grounds, both in engineering training and professional practice, for analytical methods based on elastic rigidity (stress-strain curve).

Masonry structures cannot be analyzed on such a basis: stone or brick elasticity is not readily measured. As a result, they are viewed as mere aggregates of solids working under compression only, pursuant to a statically indeterminate model of infinite order as a result of their likewise infinite number of articulated bonds. Moreover, like all structures they change with time, rendering any analytical model trivial unless account is taken of the actual conditions (fissures or cracking) prevailing in the structure at any given time of its service life.

This paper modestly attempts to clarify the assumptions on which analysis is built, evaluating the basic principles of equilibrium, the only valid grounds for such analysis, and the conditions in which such equilibrium can be reached. Yesterday's builders drew exclusively from experience to ensure equilibrium: for them structural safety meant the absence of collapse. Today vector algebra, observation with high precision instruments and a priori determination of the conditions that may lead to collapse underlie accurate and reliable conjecture about the performance of masonry structures that tangibly establishes safety levels at least as precisely as sophisticated methods of analysis. 


\section{INTRODUCCIÓN}

No se poseen datos ciertos acerca de cuando el hombre se propone construir una estructura, en el concepto que hoy conocemos de estructura mecánica, o sólido material que, construido sobre la corteza terrestre, desafía en el tiempo, a base de energía potencial, su presencia física; pero lo que parece más claro es que el hombre, en su constante búsqueda de encontrar cobijo, ha ido avanzando, a lo largo de los tiempos y en base a sus propios hallazgos -que le han ido posibilitando ser cada vez más osado en la consecución de sus logros-, hasta la situación actual.

La cronología de sus hallazgos es relativamente reciente, es decir, sólo después de la revolución francesa y los intentos de codificar el conocimiento, la Enciclopedia, la aparición del conocimiento ortodoxo ordenado en universidades, las Academias, la administración moderna y la aparición del historicismo como herramienta para valorar de una manera sistemática el pasado, sirvieron para explicar una historia del arte como, en ocasiones, nos han contado a todos, es decir, como historia de sucesos y de formas, en donde éstas se van sucediendo al albur de la imaginación de sus creadores del momento.

En el terreno de la arquitectura, los estilos clásicos de la misma (románico, gótico, renacimiento,...) han ido acompañados de fuertes descubrimientos, no sólo de la mecánica, cuanto de revoluciones sociales. La arquitectura se hace con la historia de sus pueblos, su geografía, su clima y su cultura y es el fiel reflejo de ellos mismos. Baste como botón de muestra, que, los rascacielos de los años 30 del pasado siglo, sólo fueron posibles cuando apareció el invento del cable de acero, y la empresa Otis llegó a realizar un ascensor capaz de subir ochenta pisos. Éste fue el invento que posibilitó el rascacielos, y no la aparición del acero laminado, causa ésta explicada por algunos, aun cuando el acero laminado siga teniendo un protagonismo especial en la tipología de los rascacielos.

La cultura mediterránea, de la que procedemos los pueblos que hoy constituimos Europa, está basada, en sus orígenes, en la de mesoasia, es decir, en las árabes, egipcias y mesopotámicas, que conformaron las primeras formas empíricas de hacer construcción. A estos pueblos podríamos atribuir la autoría de las primeras estructuras de fábrica, estructuras de autoconstrucción, sencillas, basadas en soluciones de subsistencia, como sus economías, pero de gran sabiduría, que, el occidente romano, sabría aprovechar y engrandecer.

Así pues, una fábrica es toda estructura mecánica (sistema nulo de fuerzas) materializada a base de agregados inertes (materiales de trabajo a monotensión como la piedra, el ladrillo, el adobe, el tapial,..) cuya única misión es pesar y mantener sus elementos comprimidos. Es el peso propio de lo que está hecha, su disposición, y sus proporciones lo que deben ordenarse para su equilibrio. Las primeras fábricas se hicieron de piedras simples desbastadas, después pasaron a realizarse de elementos cerámicos, adobes, incluso tapiales como elementos de una economía de medios elemental, para pasar, posteriormente, a realizarse de piedra, elemento, éste, de más durabilidad, aunque de trabajo más concreto, especializado y laborioso. Una fábrica es un muro, pero también una bóveda o una cúpula. La fábrica es la estructura por excelencia. Nuestro mundo "civilizado" occidental, apegado, cada vez más, de forma equívoca, al cálculo y a sus herramientas, nos ha hecho ir perdiendo la calidad sobre la visión y contemplación de los objetos de todo tipo, en especial sobre los construidos. Obsesionados por el número y cálculo, proceso que lo soporta, los técnicos intentan explicar el funcionamiento de las estructuras desde los límites del proceso de cálculo, normativas al uso y, pocas veces, desde los principios fundamentales del equilibrio y sus límites. Es baladí el intento de aproximarse cada vez más al objeto real a través del modelo de una definición científica más aproximada del mismo. El objeto muta con el tiempo y lo mismo que la viga de hormigón no es igual ahora que dentro de veinte años debido al proceso activo de fisuración, la bóveda de piedra fisurada ofrece un modelo de análisis muy distinto al de su forma completa original.

Los procedimientos para el diseño y construcción de arcos y bóvedas a lo largo de la historia son muchos, y muy diversos, dado el período tan grande de tiempo que nos ocupa, por lo que puede decirse que, todos los estilos y procedimientos están presentes en este $2^{\circ}$ milenio y de él debemos aprender con atenta lectura. Tanto el arco como la bóveda y/o cúpula, no constituyen sólo una forma más o menos grata, sino todo un procedimiento de pensar, de construir, que nos hace comprender mejor una determinada cultura.

Un edificio gótico (Fig. 1), tiene casi tres veces menos volumen de piedra y material que uno análogo romano. Esto puede no entenderse si no se comprende cómo era la sociedad medieval que lo levantó. La catedral más grande está hecha, por lo general, de sillares pequeños, manejables por un solo operario, que, además, firmará su obra (marcas de cantero) sólo con la intención de cobrar su trabajo (primera certificación de obra), hecho muy distinto a cualquier templo egipcio, griego o romano en donde la esclavitud permitió construir con grandes sillerías megalómanas, que no tanto justifican el ingenio cuanto la barbarie de sus constructores. La bóveda de Santa María dei Fiore de Florencia (Fig. 2), eterno ejemplo de proyecto y dirección de obra fue un constante quebradero de cabeza para su creador, Filipo Bruneleschi, por las constantes dificultades para contratar obreros ceramistas capaces de aparejar su bóveda, situación ésta muy distinta de las anteriores mencionadas, y que sólo intentan relacionar forma y solución estructural con cada tiempo.

Toda la historia de la arquitectura ha usado de las fábricas hasta la aparición, en el siglo XIX, de los materiales metáli- 


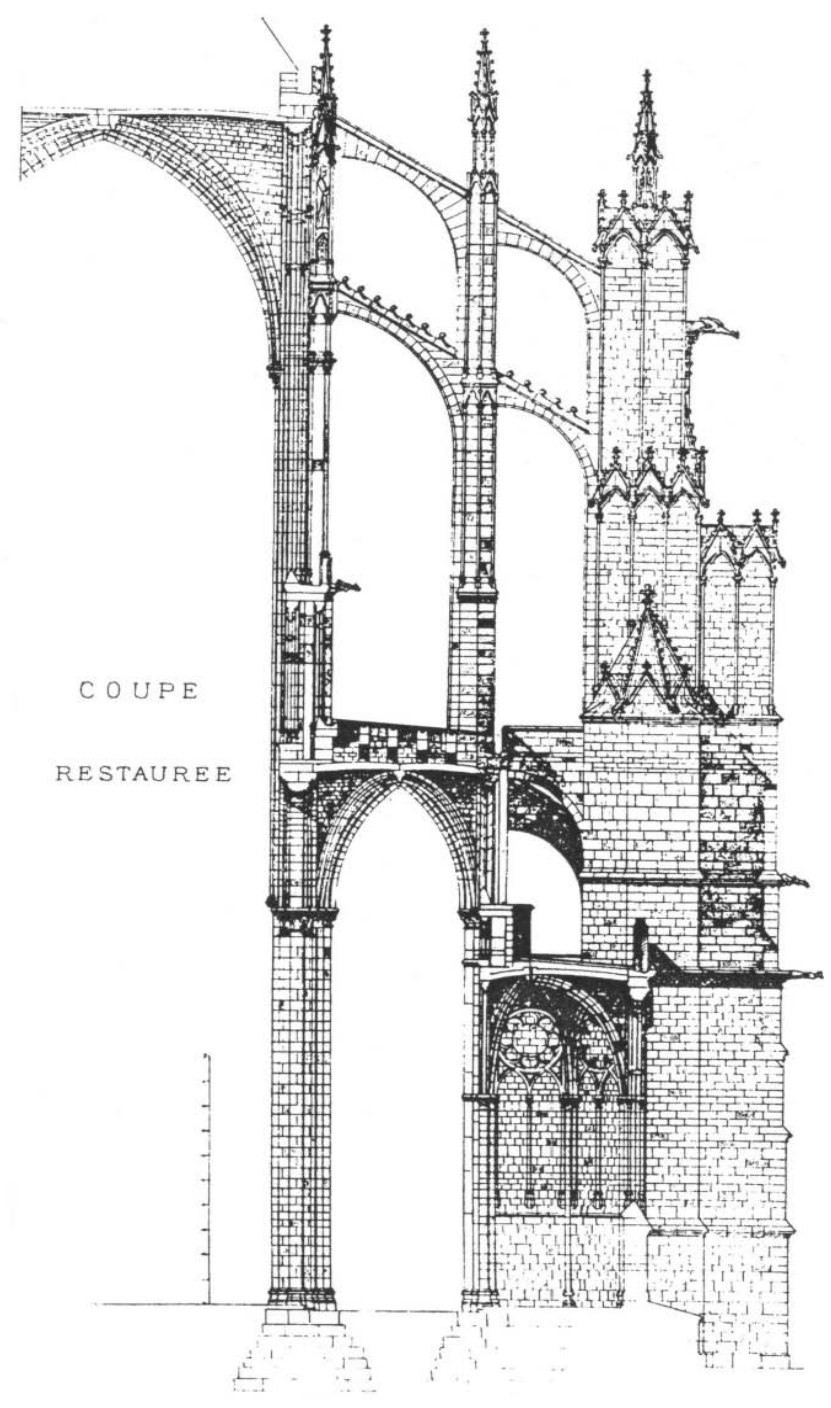

Figura 1.- Catedral de Beauvais Francia según Benouille.

cos, primero las fundiciones, después los aceros laminados y cableados. Todavía hoy, aunque sumidos en una sociedad mega-industrializada, el $60 \%$ del mundo (África, Asia y gran parte de América del Sur) sigue utilizando los materiales compresivos, ladrillo, adobe, tapial, para levantar sus edificios que, con sabiduría oriental, se posan en el suelo con una vocación de envejecer, mayor si cabe, que la de nuestros normativos y pensados edificios de hormigón armado. Todavía sigue siendo un misterio cómo las sociedades yemeníes son capaces de realizar edificios de 10-12 alturas sin más material que el adobe y el tapial, con ausencia de materiales traccionados.

La aparición en el mercado de los materiales tenaces, y la posibilidad de concentrar tensión en puntos concretos, unido al avance de los métodos de análisis y posterior cálculo, así como todo un desarrollo normativo basado en el límite numérico y la comprobación del cumplimiento de la norma, han postergado los métodos basados en la comprobación de la simple estabilidad y el equilibrio. No obs-

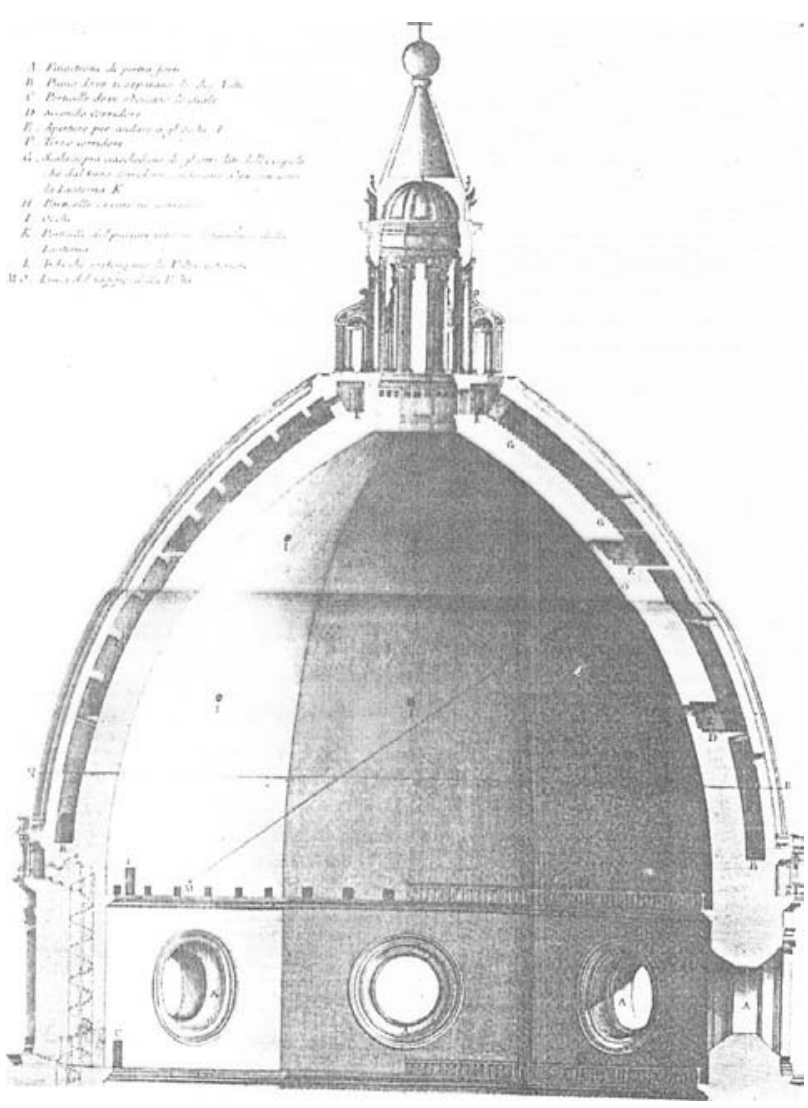

Figura 2.- Sección original de cúpula de Sta.María dei Fiore.

tante, debemos recordar que la inmensa mayoría de los hundimientos de los edificios, sucede por problemas de estabilidad y no por problemas de resistencia, aspecto, este último, que parece interesar, sólo de forma persistente, al legislador sobre estructuras, abandonándose el primero, la estabilidad, por el proyectista de estructuras.

El límite numérico y la forma estructural idónea, son dos resultados del proceso de diseño bien distintos. Utilizar el primero en estructuras de fábrica, es decir, el resultado de un cálculo numérico, prescindiendo de la inspección de la estructura y de su estado, no suele dar resultados acordes con la realidad.

\section{EL ARCO Y EL DINTEL}

La tipología de la bóveda surge como traslación de un arco (curvo o poligonal) a través de una recta. Sobre el arco se ha construido toda la historia de la arquitectura, hasta la aparición de los materiales tenaces (hierro, acero, aluminio,..), a finales del siglo XIX con la revolución industrial, y gran parte de la obra pública de este siglo (puentes, presas depósitos) se basan en este modelo, que sigue siendo el modelo mecánico que proporciona mejores alcances (tamaño máximo de la estructura sometida a peso propio). Cualquier dintel alberga dentro un arco, sólo es cuestión de verlo, de sentirlo. Gran 


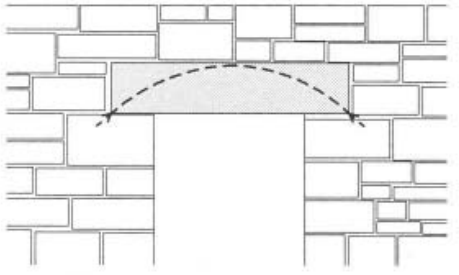

Arco. Falso dintel

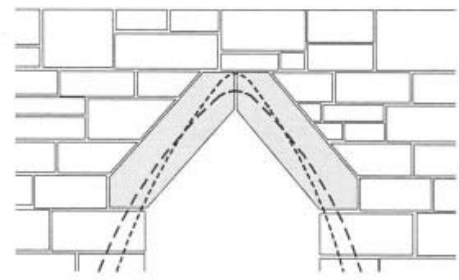

Arco maya

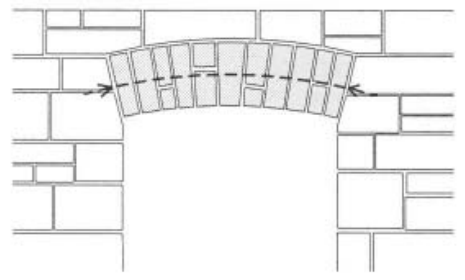

Arco romano rebajado

Figura 3.- Soluciones de huecos de muros.

parte del problema estructural está en una lúcida reflexión sobre su perfecta comprensión (Fig. 3).

En este contexto, han llegado a nuestras manos obras de la antigüedad, o no, que se rigen por el modelo del arco, o arquitectura de empujes, no en el estado original, sino deterioradas por algún motivo, que, desde aquí, no se analiza. El resultado es una estructura que se arruina. Todas las estructuras se arruinan desde su construcción, el problema es la velocidad con que lo hagan. El accidente ocasiona ruina o colapso instantáneo, el deterioro lleva a la ruina, que siempre se producirá por dos factores,

-Variación en la condición de contorno (acciones exteriores). -Cambio de fase de los materiales constitutivos (acciones interiores).

Si no existe esa variación de carga o de las propiedades del material, las estructuras permanecen en su estado de forma indefinida. Es ese valor de mutabilidad en el tiempo, lo que invalida los propios modelos y el hecho estático del objeto a analizar. Son siempre los principios de equilibrio, acción de la gravedad, capacidad de servicio de una estructura, entre otros, los únicos valores que deben de permanecer. Los métodos de análisis, o las normativas sólo son procedimientos temporales de ayuda al conocimiento y al control, reflejando en cada momento la cultura del momento, $\mathrm{o}$ ¿es que acaso los edificios del siglo XV se sostenían por causas distintas a las actuales?
En toda la historia, los arquitectos utilizaron criterios de forma y proporción en sus obras, basándose en estas dos ideas intuitivas, que entrañaban el movimiento de dos variables y que Galileo fue el primero en correlacionar, la tensión del material (dimensión cuadrática superficial), y el peso propio (dimensión cúbica volumétrica) (Fig. 4). En estructuras como las cupuliformes en donde la tensión de trabajo se utiliza casi en su totalidad en resistir el peso propio, es básica esta relación de variables, que, por otra parte, aparece en todas las formulaciones de los diferentes tipos estructurales en donde las cargas se asocian a la forma y al tamaño (peso propio) que no a la superficie de carga que soportan.

Esta relación entre el peso propio (volumen) y el material de constitución (tensión máxima que puede resistirse) ha constituido la regla de oro que limita el tamaño máximo de las estructuras.

La bóveda de S. Pedro del Vaticano o la del Panteón de Roma son dos ejemplos de tamaño máximo, y el hundimiento de la catedral de Beauvais o el de la torre Pendenti Garisenda de Bolonia son ejemplos de lo que sucede cuando se pasan los límites ordenados por el tamaño máximo que se otorga a cada material.

Toda la tratadística, que arrancó de Vitrubio hasta el siglo XIX, se construyó sobre el oficio del constructor, magíster operis, arquitecto, habilidades éstas adquiridas del propio trabajo, tan olvidadas hoy en nuestras generaciones que se refugian en la validez del número, y no en una teoría de la arquitectura basada en las proporciones, escenario éste en el que se desarrolla toda la historia de la arquitectura entendida como bella arte útil (utilitas, firmitas, venustas). Cuando Rodrigo Gil de Hontañón, en su Compendio y Simetría de Templos, a través de su discípulo Simón García nos aconseja no pasar de unas determinadas proporciones, está utilizando un sistema de prueba y error, basado en una experiencia acrisolada por un conocimiento claro de la lex artis de la construcción. Modificar esas proporciones es, sin duda, provocar la rotura o el colapso, en su caso, de la estructura. No conocía ni los vectores deslizantes, ni las ecuaciones de equilibrio, pero su sola experiencia nos ha legado el conjunto universal de bóvedas de piedra más completo que existe.

Así, se caminó hasta finales del s. XIX, con el acero y, después, con el hormigón pretensado, cuando se ha renovado el análisis estructural en los últimos años. Como dice el profesor Jacques Heyman «...Una catedral era diseñada por un master of the work» que aprendió del trabajo diario, este curso contrasta con el camino occidental basado en el concepto de «señor» arquitecto. La evolución del ingeniero tiene sus raíces en el Gótico y la del arquitecto en el Renacimiento y se institucionaliza en el segundo imperio en Francia, hacia 1835. Hasta esa fecha el arquitecto habia controlado todo el proceso de la obra.

Las acciones básicas de la naturaleza de una bóveda derivan del material, es decir, de su peso propio, principal acción en 


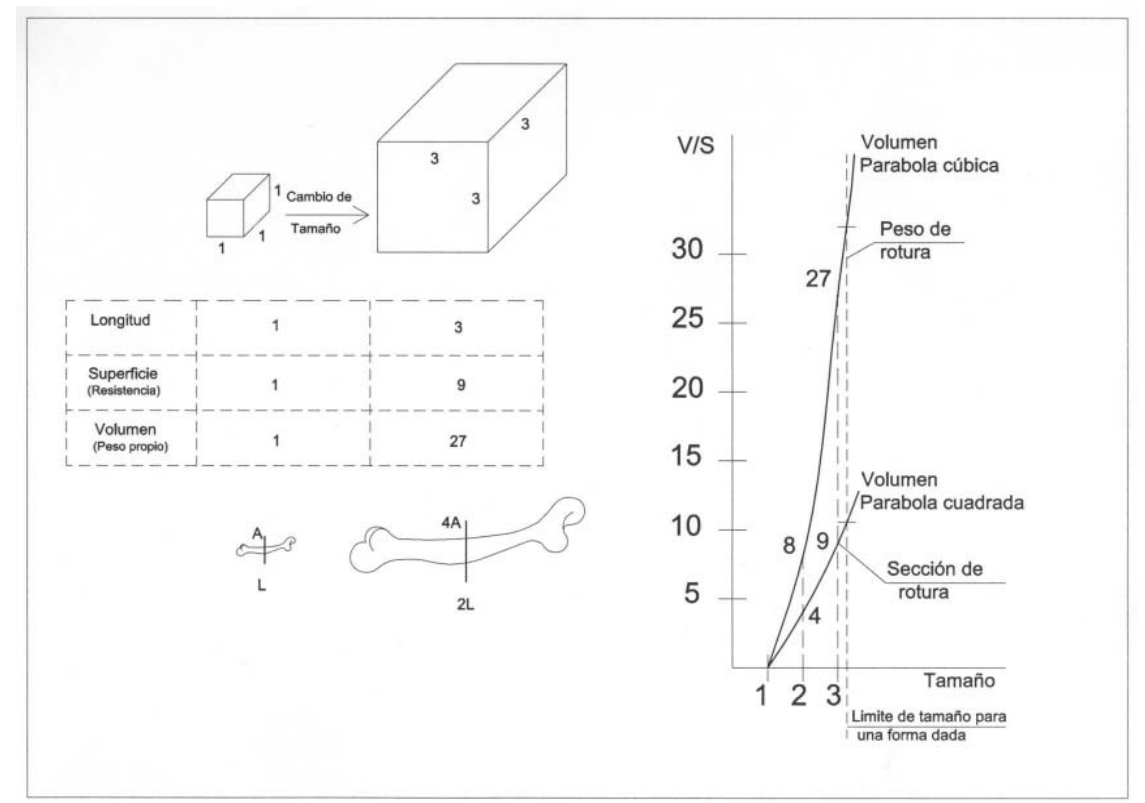

Figura 4.- Relaciones de Galileo en el crecimiento de las magnitudes, tamaño, área resistente y peso propio.

este tipo de edificios, por lo que para entender los efectos producidos por las acciones, es necesario construir una teoría estructural que incorpore las curiosas propiedades de las fábricas, puesto que el análisis clásico deja al descubierto serias incongruencias de principio, ya que se basan en unos modelos de sólido elástico basados en la continuidad. Ambos hechos en una fábrica, no se producen. Ni el material es elástico, ya que es frágil, ni es continuo, ya que la fábrica funciona multifisurada, hecho que la hace casi indefinible, por lo que hay que recurrir para su análisis a otras visiones del objeto basadas en sus situaciones límite ${ }^{1}$.

El problema, en términos de resistencia, fue planteado primeramente por Galileo, el fue el primero en considerar el análisis a resistencia de una estructura (Dos nuevas ciencias, 1638) y puso fin a la estructura medieval. El problema de Galileo en el s. XIX, dos siglos después, se fue transformado en un problema de determinación del valor de tensión. De aquí se pasó a intentar encontrar valores de tensión con adecuado margen de seguridad que, comparados con las tensiones límites de los materiales, ofrecieran soluciones aceptables.

Más tarde, Navier (1826) contestó a Galileo, diciendo que no estaba interesado en conocer el colapso de la estructura. La realidad de todo ello, sustenta el hecho de que las ecuaciones de equilibrio, aplicadas a las fábricas, no resuelven el problema, ya que éste es insoluble, la estructura de fábrica es estáticamente indeterminada (hiperestática). Baste para ello intentar construir el polígono funicular para un determinado sistema de cargas dentro de un arco de fábrica y comprobar que depende del punto de inicio de aquél para

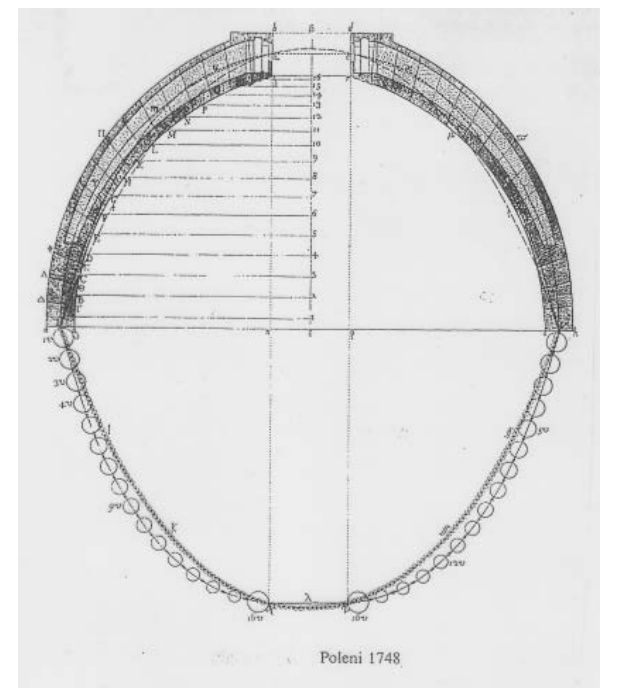

Figura 5.- Estudio de equilibrio basado en la curva catenaria de G. Poleni, 1748.

que existan infinitas soluciones, todas ellas posibles. Un arco de fábrica, como agregado de piezas talladas unidas por argamasa, es una estructura que expresa un problema con infinitos grados de libertad, por lo que no tiene solución, sin la adopción de bases de partida previas.

El arco de fábrica tiene infinitas configuraciones de equilibrio desde la cadena de Robert Hooke y el hilo cargado de La Hire (1675). La dependencia de la geometría queda expresada por Poleni (1748) en la cúpula vaticana de San Pedro (Fig. 5), cuando llamado a consulta por el Papa Alejandro XIV comprueba que se han roto tres de los tirantes de la

\footnotetext{
(1) El estudio de los modelos de fractura en los últimos años, ha supuesto un avance significativo y esencial en conocimiento del comportamiento de materiales y estructura. De una parte, por lo que supone al acercamiento del conocimiento del funcionamiento real de las estructuras, de otra, por las consecuencias normativas. La norma EHE-98, sin abandonar los postulados basados en el momento tope, impone el cálculo a fisuración como ya estaba establecido en otras normativas europeas.
} 


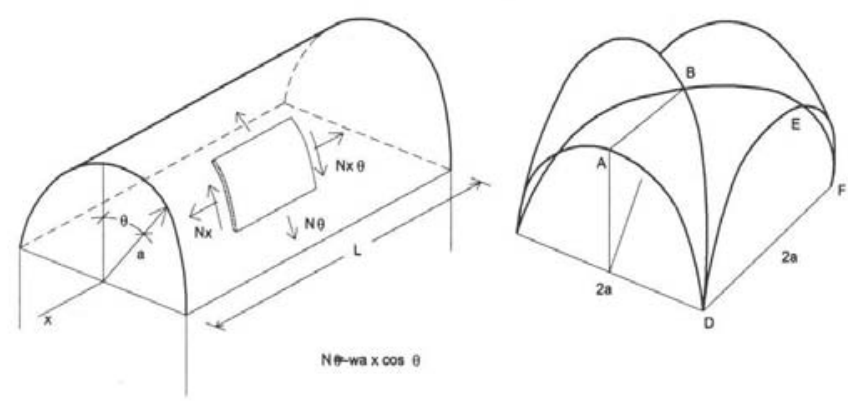

a)

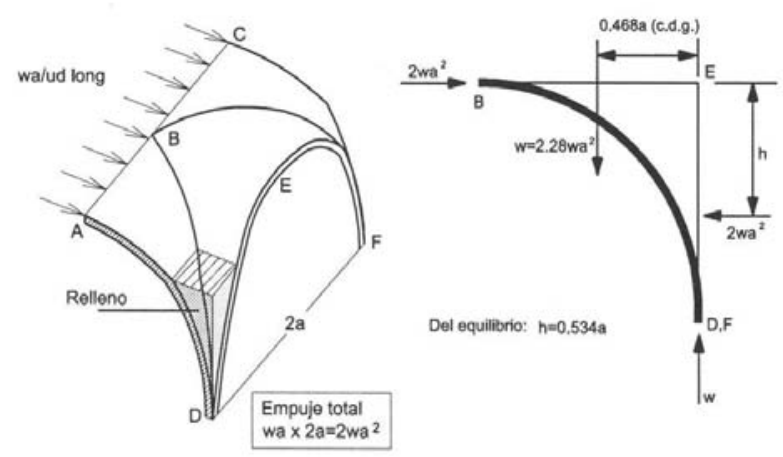

b)

Figura 6.- Localización de empujes en una bóveda, a) de medio punto y b) de crucería.

bóveda y desde su excelente Memoire Historische della gran cupola dei templo Vaticano (considerado como el primer tratado de patologia estructural) demuestra la necesidad de colocar otros seis tirantes más de $56 \mathrm{~cm}^{2}$ cada uno, que, posteriormente, construirá Vanvitelli, arquitecto conservador del Vaticano.

Después, esa bóveda ha seguido dando problemas, como en origen, consecuencia lógica de una bóveda de doble hoja hemiesférica, cargada fuertemente en punta (linterna) y con muy pocas posibilidades de responder por flexión. Casi un siglo antes, Filippo Bruneleschi hizo una bóveda de análogas dimensiones que no ha dado problemas por utilizar los principios medievales, es decir, arcos apuntados, cargados en clave y una magnífica construcción de ladrillo, a base de arcos entrelazados.

Las ecuaciones de equilibrio, por sí solas, no aportan la solución para determinar la posición del polígono de presiones, que responde a un esquema de cargas dado y debe usarse el análisis.

$1^{\circ}$ ) El material $\rightarrow$ deformaciones $\rightarrow$ fuerzas internas.

$2^{\circ}$ ) La geometría de la estructura, su forma.

Todo arco de fábrica debe ser compatible con las condiciones de contorno. El problema del arco, se basa en establecer de forma real la continuidad necesaria del contacto entre sus dovelas, que obliga a un rigor en la continuidad de su forma y a tener contenida ésta en unos límites para que su estabilidad sea posible.

Entraríamos aquí a considerar los dos tipos estructurales por excelencia con rigor mecánico, de una parte, la estructura adintelada, que ofrece respuesta por rigidez, poco sensible a la variación posicional de la carga y mucho al incremento de ella por dimensionarse a tensiones del material límites. De otra, la estructura cupuliforme o abovedada, poco sensible a la cantidad de carga, y mucho a su distribución, ya que su régimen de tensiones suele ser bajo en relación con la resistencia. Éste es el caso que ocupa a las estructuras de fábrica.

La bóveda de medio punto (Fig. 6a), utilizada sistemáticamente por los romanos, es una estructura mucho más estable que la sucesión de arcos separados que la conforman. El rozamiento entre dovelas y la traba juegan un papel primordial. La traba justifica la adopción de mecanismos secundarios que pueden desfigurar el modelo ideal resistente. Es fácil encontrar arcos y bóvedas con más grietas de las que el modelo permite para ser considerado estructura y no mecanismo. La razón de su existencia está más en el rozamiento y en la colaboración de la concarga del arco, o carga pasiva del arco/ bóveda que colabora en coaccionar movimientos y estabilizar el conjunto. Para que una estructura colapse, tiene que poder hacerlo, es decir, tiene que poder ejecutar movimientos cinemáticamente admisibles que la conviertan en mecanismo, si esto no puede suceder, la estructura autotransforma su sistema de respuesta (polígono de presiones) en otro distinto. Es esencial obtener esta aproximación y situar correctamente las líneas de fuerza nuevas para poder peritar la estructura.

La bóveda de crucería gótica (Fig. 6b), forma un conjunto de mucha más trascendencia como modelo, por lo que significa, sin utilizar materiales tenaces, el mismo corte de sillar para todos los arcos, esfuerzos dirigidos a puntos concretos, diafanidad de muros,...etc. En este sentido, si se observa el último Gótico (Milán, Ackerman 1949), «la estructura juega un rol secundario en el proceso creativo», ya que conforme nos adentramos en el renacimiento se va perdiendo pureza en el planteamiento, en aras del color o la proporción usada en términos de armonía (arco perfecto circular) y no de mecánica.

Una cúpula puede generarse por el entronque de una o varias bóvedas de cañón o por rotación de una forma curva o poligonal en torno a un eje, generalmente vertical (cúpula). El análisis elástico extraído de la teoría de la membrana, no es exacto para superficies en las que el canto es relevante. $\mathrm{Si}$ comparamos la luz (L) al canto (t), como hace Heyman:

$\mathrm{L}(\mathrm{Luz}) \quad \mathrm{t}(\mathrm{mm}) \quad \mathrm{L} / \mathrm{t}$

$\begin{array}{lccc}\text { San Pedro Vaticano } & 42,5 & 3.000 & 14 \\ \text { Cáscara de huevo } & 0,04 & 0,4 & 100 \\ \text { Mercado Smithfield } & 68 & 75 & 900\end{array}$


La rigidez de la cáscara de huevo evidencia este hecho. Si a esto se añade que las cúpulas por razón de forma se encuentran sometidas a estados de tracción y los materiales que conforman las bóvedas de fábrica no resisten a tracción, concluiremos que las bóvedas, para trabajar, han de estar fisuradas. Prescindir de esta realidad puede suponer no entrar ni siquiera en la comprensión del funcionamiento de una estructura de fábrica.

Si los criterios para considerar una estructura como viable, se basan en las tres cualidades estructurales, resistencia, rigidez y estabilidad, como básicas, se comprende que, en las bóvedas, estos requerimientos no operan igual. Si estos tres criterios pueden ser satisfechos, la estructura está en servicio. En un arco exento supone satisfacer las tres cuestiones de la forma:

- resistencia $(\alpha)$ : el arco no romperá, ya que las tensiones son bajas en relación con las de rotura de la fábrica,

. rigidez: los movimientos o traslaciones (nunca flechas elásticas) serán tolerables,

. estabilidad: nunca se formarán 4 rótulas (mecanismo).

La solución elástica del problema no resuelve las condiciones de rigidez de apoyo (condición de contorno), por lo que una bóveda está siempre buscando su equilibrio en la pérdida de energía potencial, que puede deberse a muchas causas. Un suelo de roca es rígido, pero todos los demás no, y las bóvedas se apoyan en pilares.

La teoría plástica nos dice que si dos estructuras, aparentemente idénticas, con diferentes imperfecciones y diferentes estados de tensión inicial son cargadas despacio hasta el colapso, se encontrará la misma carga de colapso para las dos.

Esta observación general del desarrollo de la teoría plástica no es aplicable al caso de colapso dúctil. El cálculo se basa en un hipotético valor de carga incrementada por un factor. En el curso del cálculo del hipotético colapso el diseño plástico genera un estado de equilibrio para un estado real de cargas de trabajo.

Los diseñadores de estructuras por métodos elásticos creen que han generado el estado de equilibrio, mientras que los diseñadores plásticos saben que sólo han generado un particular estado de los infinitos posibles de equilibrio. Ya que para estos últimos, todo el problema consiste en encontrar un estado de equilibrio tal, que si unas fuerzas internas están en equilibrio con las cargas externas y, además, con todas las partes, satisfacen los criterios de resistencia, la estructura es estable. Es obvio que hay que buscar aquéllas que tengan un adecuado margen de seguridad.

Acometer un problema de patología de bóvedas desde la óptica plástica sería algo así como un diagnóstico médico que concluyera con que el enfermo moriría al llegar a los $43^{\circ}$ de temperatura, es decir, establecer un límite, hecho que al paciente puede importar poco y más la forma de acercarse a esa temperatura, es decir, el proceso evolutivo de su enfermedad y sus consecuencias.

Determinar la posible carga de colapso de una estructura implica desconocer, porque está implícito en el proceso, la historia del mismo, historia fisurativa clave en el análisis elástico y en las soluciones, ya que siempre hablamos de estructuras con grandes imperfecciones de tipo constructivo que se apartan del modelo. Toda la ingeniería francesa del s. XIX y la sofisticada analítica del XX, han trazado sus objetivos en describir con perfección la historia del proceso resistente y deformacional, el scanner en medicina, el cálculo matricial, el método de elementos finitos o de elementos de contorno en mecánica son buenos ejemplos de ello, sin pensar que el propio objeto del estudio, no es el estudio es si mismo, cuanto la descripción suficiente para gobernar los problemas, con una credibilidad que justifique las soluciones. Sin menospreciar para nada el fabuloso y eficaz desarrollo de los métodos de análisis y comprobación recientes, derivados, en su mayoría, del uso del ordenador y su capacidad de aproximar, totalmente indicados y contrastados para estructuras de acero u hormigón, las estructuras de fábrica requieren otra visión, menos exacta, si se quiere, pero más sabia y comprensiva del fenómeno del arco de fábrica puesto en carga y de la nueva situación de equilibrio tras la fisuración, problema, éste, clave, olvidado durante siglos y sin cuya solución no puede describirse el estado real de la estructura.

Este hecho lleva a plantearse el problema de la reparación de cúpulas y bóvedas partiendo del modelo geométrico fisurado real. Estas estructuras se construyeron para una forma. Los estados de agrietamiento y fisuración no implican ruina, sino solo que la estructura esta trabajando de forma distinta.

En toda fábrica partiremos de tres supuestos:

- no hay resistencia a tracción,

- las tensiones son pequeñas,

- la rotura por corrimiento no existe.

Los modelos están basados en la geometría. Galileo fue claro en esto, demostró la relación escala (peso, densidad), resistencia (fuerza de resistencia por unidad de área). Para un diseño (forma) y un material $(\alpha)$ hay un tamaño máximo.

$\begin{array}{lcl}\text { Santa Sofía, año 532 } & \text { luz } & 31,0 \mathrm{~m} \\ \text { San Pedro, 1560 } & \text { luz } & 42,5 \mathrm{~m} \text { con tambor } \\ \text { Santa María, 1420 } & \text { luz } & 36,0 \mathrm{~m} \text { con tambor } \\ \text { Panteón, 120 } & \text { luz } & 43,0 \mathrm{~m}\end{array}$

La asimilación de la teoría de la membrana en una bóveda no es exactamente correcta pues implica que no va a haber otros esfuerzos que no sean en su plano, y que el espesor es despreciable frente a otras dimensiones.

La cadena de Hooke no tiene rigidez y el arco es muy sensible a la variación de forma. Eso no sucede si la estructura tiene 


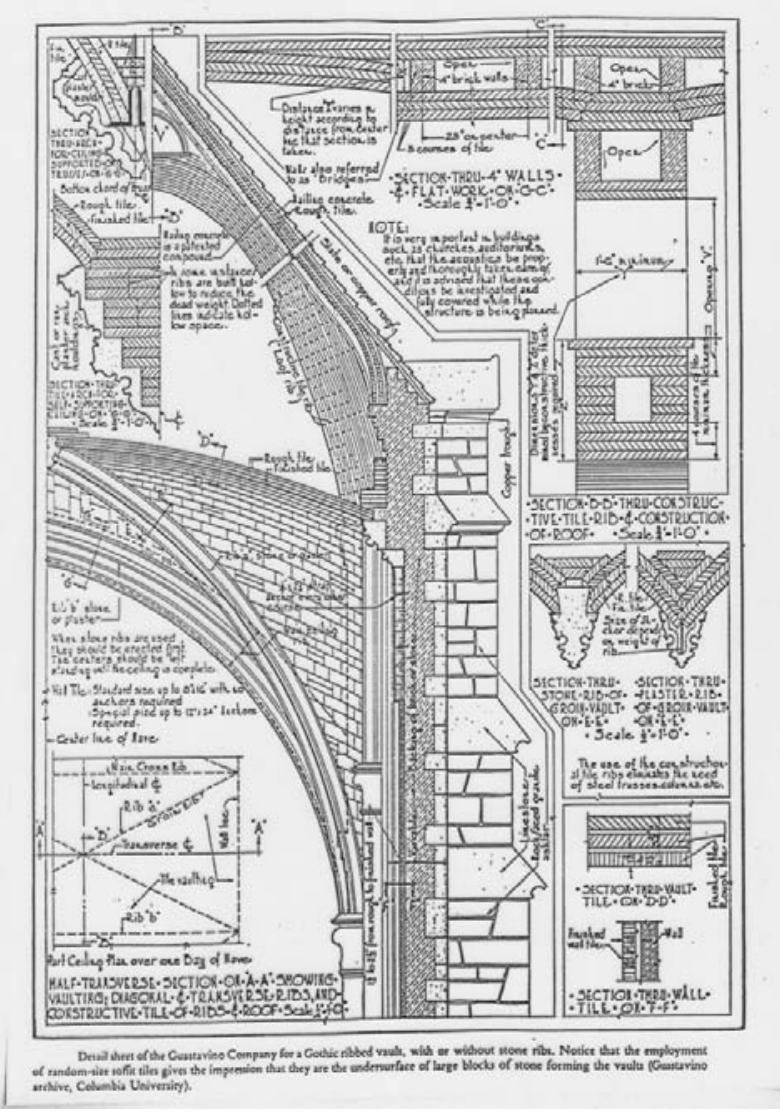

Figura 7.- Detalles de la Guastavino Compañy para la Universidad de Colombia, publicados en el cátalago de su exposición. Madrid, 1999.

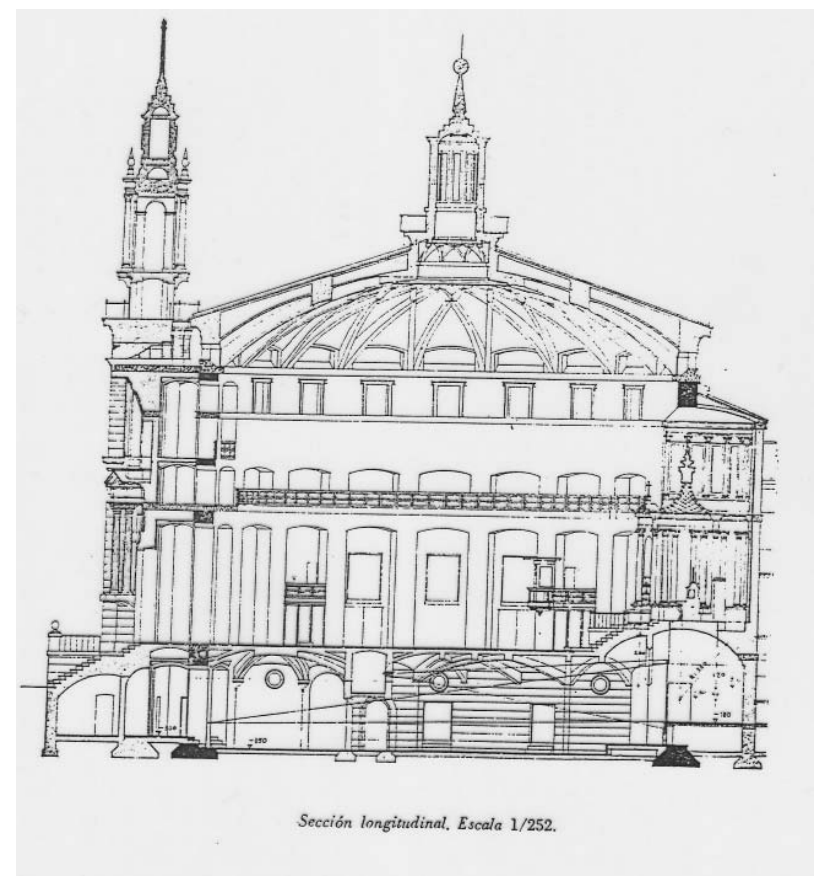

Figura 8.- Luis Moya Iglesia de S. Agustín. Sección (de su libro, Bóvedas Tabicadas). además doble curvatura y/o rozamiento, ya que tiene otras posibles formas de evacuar la carga.

En toda estructura siempre hay fisuras que expresan la búsqueda de un sistema propio de sostenerse algo distinto al pensado originalmente. Todo el análisis está basado en esta consideración. Como ejemplo puede citarse el edificio de Santa Sofía (Estambul), estructura compleja que sufrió dos sísmos (en 986 y en 1346), estos accidentes hicieron fracasar la bóveda dos veces. Desde el siglo VI de su construcción hasta hoy ha tenido una constante degradación de su bóveda $\mathrm{y}$, como consecuencia, de todo el edificio.

Sólo el conocimiento de la estructura real (fisurada) puede aportar datos de comportamiento, sobre el que establecer las bases de un análisis y un diagnóstico plausibles.

Esta concepción estructural de la fábrica puede olvidar todas las aportaciones realizadas en el siglo XVI, XVIII, XIX, por Alberti, La Hire, Polen, Frezier, Danyzy, Coulomb, Barlow W.H., Belidor, Blondel, Fray Fco. Cabezas, y tantos otros, a través de la tratadística y del aporte cinético concreto han permitido que conozcamos hoy, un poco mejor, las estructuras de fábrica. En nuestros días, el profesor inglés Jacques Heyman ha sido clave para este conocimiento.

En el siglo XIX, Gaudí con sus bóvedas extraídas directamente de los modelos catenarios, o el español Rafael Guastavino (Fig. 7) y su hijo, que realizaron uno de los mejores conjuntos de construcciones de fábricas de gravedad y atirantadas tanto en Cataluña como en la emergente sociedad de Estados Unidos y es posible, que, si el primero es sobradamente conocido y estudiado, en el caso de los segundos, su obra no esté suficientemente divulgada.

El también español Luis Moya (Fig. 8), aportó a las estructuras de fábrica un denso magisterio expresado en magnificas obras para la historia de la arquitectura, su bóveda de la iglesia de la U.L. de Gijón muestra, con sólo un metro menos de luz que la bóveda miguelangesca del Vaticano, los recursos de un arte sencillo que se expresa a través de oficio aprendido.

También, aunque poco divulgada su obra, nuestro contemporáneo y recientemente desaparecido Eladio Dieste, ingeniero uruguayo, aportó la novedad de diseños de fábricas de grandes dimensiones gracias a la utilización de la fábrica armada pretensada o "cerámica estructural". Un recurso nuevo en herramienta de nuestro tiempo.

Estos tres exponentes recientes demuestran la actualidad de la mecánica de las fábricas, aparentemente amenazada por la dificultad constructiva y el obligado aporte de mano de obra, pero que justificamos, más que por una falta de conocimiento del oficio, que un día llevó a los hombre a levantar las construcciones más arriesgadas y bellas que han llegado a nuestros días, con el solo arte de poner una piedra encima de la otra. 


\section{BIBLIOGRAFÍA}

- Adam, Jean-Pierre. La Construction Romaine. Matériaux et Techniques. Editorial A. et J. Picard, Paris, 1989.

- Armenákas, Anthony E. Modern Structural Analysis. The Matrix Method Approach. Editorial Mc Graw-Hill, Inc. Nueva York, 1991

- Arnold, Dieter. Building in Egypt. Pharaonic Stone Masonry. Editorial Oxford University, Oxford, 1991.

- Bails, Benito. De la arquitectura civil. Edita: Colegio Oficial de Aparejadores y Arquitectos Técnicos de Murcia. Valencia, 1983.

- Binding, Günther. Das Dachwerkauf Kirchen im deutschen sprachraum vom Mittelalter bis zum 18. Jahrhundert. Editorial Deutscher Kunstverlag, Munich, 1991.

- Bellini, Amedeo. Teniche della Conservazione. Editorial Franco Angeli, Milán, 1990.

- Boscotrecase, L. y A. Di Tommaso. Statica Applicata alle Construzioni. Editorial Pàtron, Bolonia, 1983.

- Bruschi, A., C.L. Frommel, F.G. Wolff Metternich, C. Thoenes. San Pietro che non c'è, da Bamante a Sangallo il Giovane. Editorial Electa, Milán, 1996.

- Buttgereit, Dieter, Rolf Koschade, Werner Roswandowitsch. Gerüste. Editorial Ernst \& Sohn, Plettenberg, 1991.

- Calabro, Mateo. Tratado de fortificación o arquitectura militar. Ediciones Universidad de Salamanca, Salamanca, 1991.

- Calladine, C. R. (Ed.) Masonry Construction. Structural Mechanics and other Aspects. Editorial Kluwer Academic Publishers, Dordrecht, 1992.

- Callebat, Louis y Philippe Fleury (Eds.). Dictionnaire des termes techniques $d u$ «De architectura» de Vitruve. Editorial Olms-Weidmann Hildesheim, 1995.

- Casas Gómez, Antonio de las, Santiago Huerta Fernández y Enrique Rabasa Días. Actas del Primer Congreso de Historia de la Construcción. Edita: Instituto Juan de Herrera y CEHOPU, Madrid, 1996.

- Castigliano, C. A. P. Théorie de l'équilibre des systèmes élastiques et ses applications. Editorial Dover, Nueva York, 1966.

- Choisy, Auguste. Historia de la Arquitectura. Volumen I, Prehistoria y Antigüedad. Volumen II, Arte Bizantino y Musulmán - Edad Media - Renacimiento - Tiempos Modernos. Editorial Víctor Leru, Buenos Aires, 1970.

- Choisy, Auguste. El arte de construir en Roma. Edita: Instituto Juan de Herrera y CEHOPU. Madrid, 1999.

- Crouch, Dora P. History of Architecture. Stonehenge to Skyscrapers. Editorial Mc Graw-Hill, Inc. Nueva York, 1985.

- Dieste, Eladio. Cáscaras Autoportantes de Directriz Catenaria sin Tímpanos. Ediciones de la Banda Oriental; Montevideo, 1985 (Bibl. IETcc).

- Dieste, Eladio. La Estructura Cerámica; Facultad de Arquitectura, Universidad de los Andes (Colombia) (Colección Somo Sur); Bogotá, 1987.

- Dupuit. Traité de l'équilibre des voûtes et de la construction des ponts en maçonnerie. Dunod Editor, Paris, 1870.

- «Filarete» Averlino, Antonio. Tratado de Arquitectura. Edita: Instituto de Estudios Iconográficos EPHIALTE del Ayuntamiento de Vitoria-Gasteiz, Alibarra, 1990.

- Flugge, Wilhelm. Stresses in Shells. Editorial Springer-Verlag, Berlin, 1990.

- Francia, Ennio. Storia della costruzione del nuovo San Pietro da Michelangelo a Barnini. Editorial De Luca Edizioni D'Arte. Roma, 1989.
- García, Simón. Compendio de Arquitectura. Imprime: SeverCuesta. Valladolid, 1991.

- Gordon, J. E. The new science of strong materials or why you don't fall through the floor. Editorial Penguin Books, Londres, 1991.

- Heyman, J. The Stone Skeleton. International Journal of Solids and Structures, Vol. 2, pg. 249-279. 1966.

- Heyman, J. Teoría, Historia y Restauración de Estructuras de Fábrica (recopilación de artículos). Instituto Juan de Herrera \& CEHOPU.Madrid, 1995. (Bibl. ETSAM)

- Heyman, J. The Safety of Masonry Arches. International Journal of Mechanical Sciences, Vol. 11, pg. 363-385. 1969.

- Heyman, J. The Estimation of the Strength of Masonry Arches. Proceedings of the Institution of Civil Engineers, Vol 69, pg. 921-937. 1980.

- Heyman, J. Poleni's Problem. Proceedings of the Institution of Civil Engineers, Vol. 84, pg. 737-759. 1988.

- Heyman, J. Structural Analysis: A Historical Approach. Cambridge University Press. pg. 174. 1998.

- Huerta, S. Diseño estructural de arcos, bóvedas y cúpulas en España, ca. 1500-1800. Tésis doctoral, Escuela Técnica Superior de Arquitectura, Universidad Politécnica de Madrid. 1990.

- Johnson, Claes. Numerical solution of partial differential equations by the finite element method. Editorial Cambridge University Press, Suecia, 1992.

- La Hire, P. Sur la construction des voûtes dans les édifices. Mémoires de l'Académie Royale des Sciences de Paris. Paris, 1712 .

- Livesley, R.K. Limit analysis of structures formed from rigid blocks. International Journal for Numerical Methods in Engineering, Vol. 12, pg. 1853-1871. 1978.

- Lombardo, Salvatore y Salvatore Luparello. Restauro Strutturale 1. Editorial Dario Flaccovio, Palermo, 1997.

- Marino, Luigi. Materiali da Costruzione eTecniche Edili Antiche. Indagini e Rilievi Nell'ottica della Conservazione. Editorial Alinea, Florencia, 1991.

- Mark, Robert. Light, Wind and Structure. The Mystery of the Master Buildres. Editorial The MIT Press, Cambridge, 1990.

- Martínez de Aranda, Ginés. Cerramientos y trazas de montea. Servicio Histórico Militar, Comisión de Estudios Históricos de Obras Públicas y Urbanismo. CEHOPU, Madrid, 1986.

- Mastrodicasa, Sisto. Dissesti Statici delle Strutture Edilizie Diagnosi e Consolidamento. Editorial Ulrico Hoepli, Milán, 1973.

- Mas-Guindal, A-J. «Rehabilitación» (Serie de 9 tomos. Dirección $n^{\circ} 5$ la Estructura). COAM, 1984.

- Mas-Guindal, Sáenz de Oiza, Moya, y Otros. "Mecánica y Tecnología de los Edificios Antiguos”. COAM, 1987.

- Mas-Guindal, A-J. "Acueducto de Segovia. Un Método para el diagnóstico de las construcciones». Ministerio de Cultura, León, 1992.

- Mas-Guindal, A-J. «Las Grietas en las estructuras de fábrica. Un procedimiento para evaluar la forma de trabajo de éstas. Informes de la Construcción, Vol. 48, $\mathrm{n}^{\circ}$ 446. IETcc (C.S.I.C).

- Mas-Guindal, A-J. «Criterios de intervención y recomendaciones de diseño a sismo en las estructuras de Patrimonio Histórico. Informes de la Construcción, Vol . 48, $\mathrm{n}^{\circ}$ 443. IETcc (C.S.I.C.) - Mas-Guindal, A-J. «La Reparación de la Estructura» Fundación Cultural COAM. 1998.

- Mas-Guindal, A-J. "Procedimientos y Técnicas constructivas del Patrimonio" Universidad de Alcalá de Henares 1998. 
- Monasterio. Nueva teórica sobre el empuje de bóvedas. Manuscrito sin publicar, Biblioteca de la Escuela Técnica Superior de Ingenieros de Caminos, Canales y Puertos. Universidad Politécnica de Madrid, 1800.

- Moseley, H. The Mechanical Principles of Engineering and Architecture. Editorial Longham, Londres, 1843.

- Moya Blanco, Luis. Bóvedas tabicadas. Edita: Ministerio de la Gobernación, Dirección General de Arquitectura. Madrid, 1947.

- Muller, Werner. Grundlagen gotischer Bautechnik. Editorial Deutscher Kunstverlag, Munich, 1990.

- Navier, C. L. M. H. Résumé des leçons données à l'École des Ponts et Chaussées, sur l'application de la mécanique à l'établissement des construction et des machines. Paris, 1826.

- O'Connor, Colin. Roman Bridges. Editorial Cambridge University Press, Londres, 1993.

- Paxton, Roland. 100 Years of the Forth Bridge.Editorial Thomas Telford, Londres, 1990.

- Pearce, Martin y Richard Jobson. Bridge Builders. Editorial Wiley-Academy, Londres, 2002.
- Rankine, W. J. Manual of Applied Mechanics. Editorial Charles Griffin, Londres, 1858.

- Rodríguez Martín, Luis Felipe; Fábrica de Bloques; UNED, Escuela de la Edificación; Madrid, 1986.

- Sanabria, S. L. The Mechanization of Design in the $16^{\text {th }}$ Century: The Structural Formulae of Rodrigo Gil de Hontañón. Journal of the Society of Architectural Historians. 1982.

- San Nicolás, Fray Laurencio de. Arte y Vso de Architectvra. Albatros Ediciones, Madrid, 1989.- Torroja Miret, Eduardo. Razón y ser de los tipos estructurales. Editorial EBCOMP, S. A. Madrid, 1998.

- Viollet-Le-Duc, E. Dictionnaire Raisonné de l'Architecture Française, Vol. 2, Construction. Paris, 1854.

- Viollet-Le-Duc, E. Entretiens sur l'architecture. Pierre Maradaga, Editor. Paris, 1986.

- Viollet-Le-Duc, E. Lectures on Architecture. Vol I y Vol II. Editorial Dover Publications, Inc. Nueva York, 1987.

- Ware, Dora y Betty Beatty. Diccionario manual ilustrado de arquitectura. Ediciones G. Gili, S. A. México, 1993. 